\title{
A case report of pseudo-progression after pembrolizumab in metastatic gastric cancer and a review of immunotherapy in gastroesophageal tumors
}

\author{
Hossein Taghizadeh · Katharina Lampichler · Andrea Beer · Matthias Preusser · Aysegul Ilhan-Mutlu
}

Received: 2 August 2018 / Accepted: 7 November 2018 / Published online: 19 November 2018

(C) The Author(s) 2018

\begin{abstract}
Summary In this report, we present the medical history of a 30-year-old male patient with HER2- and PD-L1-negative metastasized adenocarcinoma of the gastric cardia, who received three cycles of pembrolizumab (200 mg every 2 weeks) after the failure of the first-line (1L) treatment with docetaxel, cisplatin, 5-fluorouracil (DCF). A restaging computed tomography (CT) scan for the chest and abdomen revealed an apparent progressive disease; therefore, the treatment was terminated. Five months after the termination of the treatment, a new CT scan demonstrated a spontaneous treatment response although no treatment was given during this time period, indicating pseudo-progression of the tumor in the first restaging after three cycles of pembrolizumab. This finding is apparently due to the long-term sustainable immunological effects of pembrolizumab. The current report will present this rare case in more detail and summarize the closed and ongoing clinical trials of immunotherapy drugs in gastroesophageal cancer.
\end{abstract}

\footnotetext{
H. Taghizadeh $\cdot$ M. Preusser $\cdot$ A. Ilhan-Mutlu, MD, PhD $(\bowtie)$ Department of Medicine I, Clinical Division of Oncology, Medical University of Vienna, Waehringer Guertel 18-20, 1090 Vienna, Austria aysegul.ilhan@meduniwien.ac.at

H. Taghizadeh · A. Beer - M. Preusser - A. Ilhan-Mutlu, MD, $\mathrm{PhD}$

Upper Gastrointestinal Tumours Unit, Comprehensive Cancer Center Vienna, Vienna, Austria

\section{K. Lampichler}

Department of Radiology, Medical University of Vienna, Vienna, Austria

\section{A. Beer}

Department of Pathology, Medical University of Vienna, Vienna, Austria
}

Keywords Gastric · Gastroesophageal · Checkpoint inhibitor · Pembrolizumab · Clinical trials

\section{Case report}

A 30-year-old male student presented to a general practitioner with weight loss and mildly reduced general condition in June 2016. A comprehensive diagnostic work-up including computed tomography (CT) of the chest and abdomen and gastroscopic biopsies revealed stage IV HER2-negative moderately differentiated (G2) tubulopapillary adenocarcinoma of the gastric cardia with multiple synchronous metastases in the lung, liver, and the lymph nodes near the truncus coeliacus and the kidneys. The family history showed that his mother died of pancreatic cancer at 52 years of age, his father died of prostate cancer at 55 years of age, and his uncle was diagnosed with sarcoma at 45 years of age. He was a smoker with a cumulative nicotine consumption of three pack-years. At the time of the disease onset, he had an excellent general condition with an Eastern Cooperative Oncology Group (ECOG) score of 0.

The patient received six cycles of palliative chemotherapy with docetaxel, cisplatin, 5-fluorouracil (DCF) from June 2016 to October 2016 in a local hospital in Vienna. Although a partial response was seen after three cycles, the final CT scan exhibited progressive disease. Consequently, the hospital changed the treatment to pembrolizumab, which was given for three cycles every 3 weeks with a dose of $200 \mathrm{mg}$ until January 2017. A subsequent restaging CT showed progressive disease; therefore, pembrolizumab was discontinued and no alternative treatment was given. Five months after discontinuation of the pembrolizumab treatment (June 2017), a new staging CT showed significant shrinkage of the metastases in Virchow's triad (Fig. 1a, b), lung (Fig. 2a, b), liver (not shown), and 

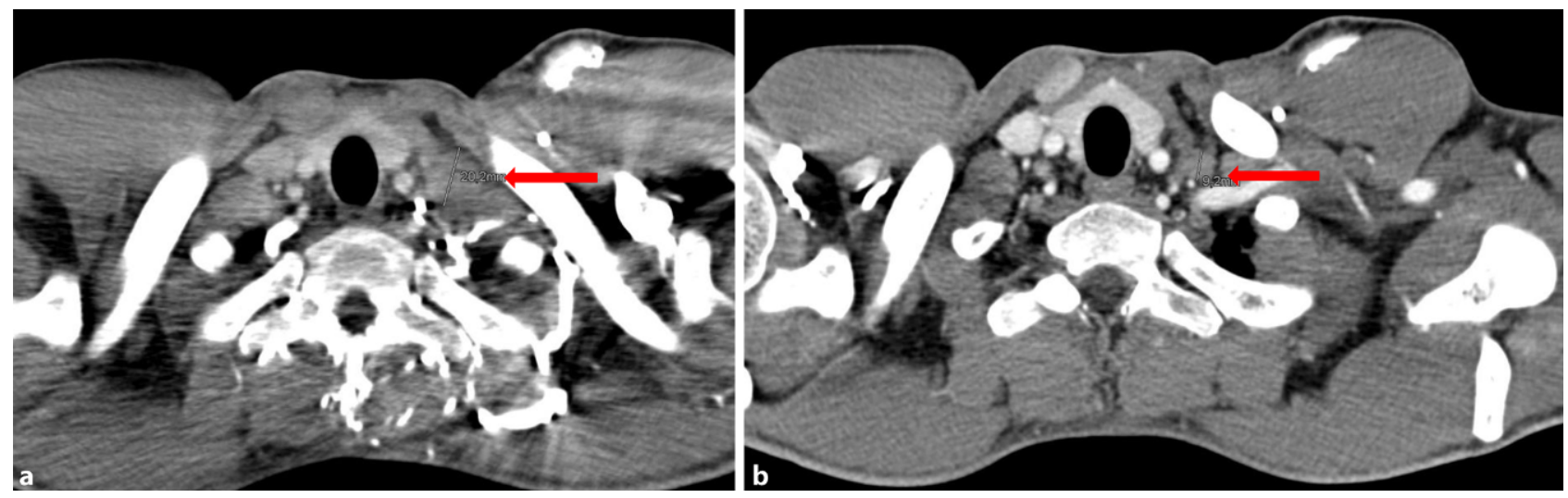

Fig. 1 Virchow's triad: a March 2017, b June 2017
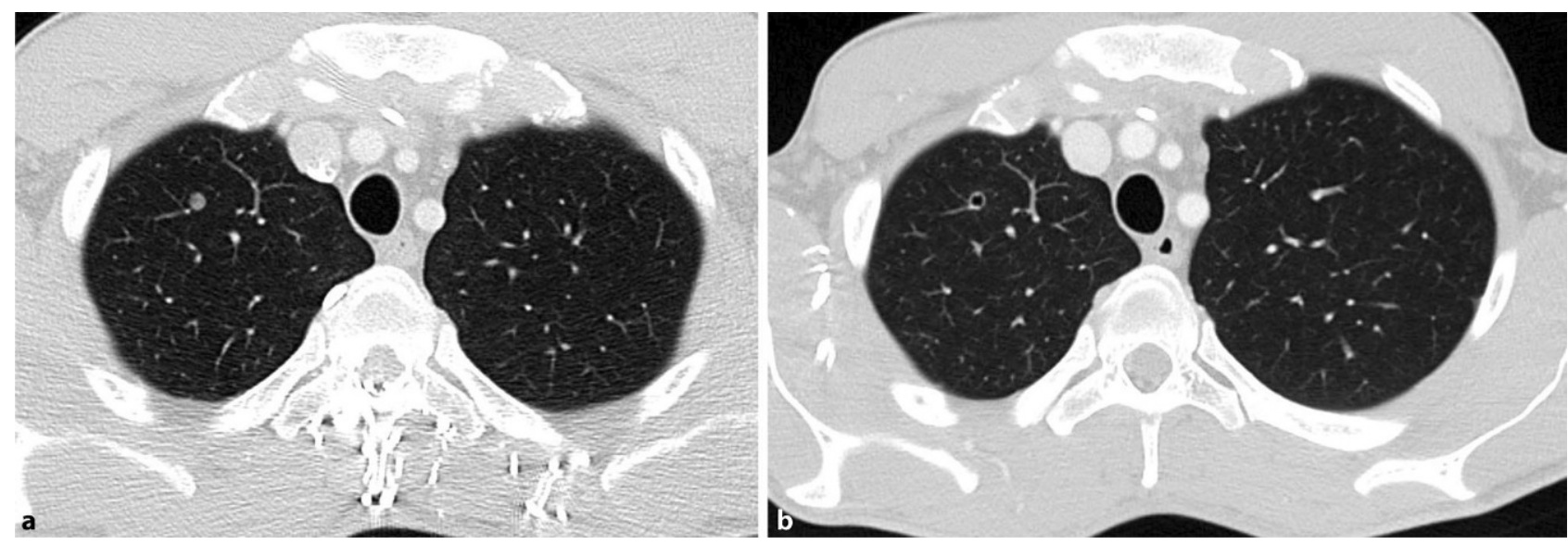

Fig. 2 Pulmonary metastasis: a March 2017, b June 2017

lymph nodes (not shown). This spontaneous regression was remarkable, since the patient did not receive any treatment during this period.

He was then referred to our specialized upper gastrointestinal (GI) tumor service for disease evaluation and possible treatment suggestions. We recommended a further "wait and see" strategy and requested a new CT scan in 3 months. The followup CT scan in November 2017 demonstrated new metastasis in the adrenal glands and enlargement of the metastasis in the lymph nodes (especially in the para-aortal region) and in the lung. Therefore, pembrolizumab therapy was reinitiated in November 2017. The restaging CT after three cycles of pembrolizumab in January 2018 revealed a mixed response with significant regression of the metastases in the lymph nodes and adrenal glands and minimal progression of the pulmonary metastasis. Further evaluation with a CT scan was performed after continuation of treatment with pembrolizumab in April 2018, which again showed a mixed response in terms of a minimal enlargement of the lymph nodes in the para-aortal region, with a reduction of other lymph nodes in the para-gastric region and two small $(<1 \mathrm{~cm})$ suspicious regions in the liver.

The tumor biopsy tissue of the patient was investigated for PD-L1 expression, which was negative for tumor cells and $1 \%$ positive for tissue-infiltrating lymphocytes. Further, a strong expression of the DNA repair enzymes MLH1, MSH2, MSH6, and PMS2 was detected both in tumor and healthy tissue, which was indicative of microsatellite stability. The patient still has a good performance status and tolerates the treatment very well. The laboratory values, including thyroid hormone levels, are in the normal range. Based on the result of the last CT scan, treatment with pembrolizumab is being continued. This study was approved by the local ethics committee.

\section{Discussion of the case report}

Enlargement of a metastatic region of the primary tumor in the CT scans is interpreted as progressive disease according to the RECIST criteria, where a treatment change is usually recommended [1]. Immune checkpoint inhibitors might enhance the accumulation of inflammatory cells, which may translate into enlargement of a target lesion. To overcome this problem, which is usually called "pseudo-progression," the RECIST criteria were adapted (iRECIST; [2]). According to the new iRECIST criteria, progression of a le- 
Table 1 Immune checkpoint inhibitors: overview

\begin{tabular}{l|l|l|l|l|l|}
\hline Substance name & Brand name & Substance number & Company & Antibody type & Target \\
\hline Pembrolizumab & Keytruda ${ }^{\circledR}$ & MK-3475 & Merck \& Co & IgG4-humanized & PD-1 \\
\hline Nivolumab & Opdivo ${ }^{\circledR}$ & BMS-936558 & Bristol-Myers Squibb & IgG4-human & PD-1 \\
\hline Ipilimumab & Yervoy ${ }^{\circledR}$ & MDX-010 & Bristol-Myers Squibb & IgG1k-human & CTLA-4 \\
\hline Durvalumab & Imfinzi ${ }^{\circledR}$ & MEDI4736 & Medimmune/AstraZeneca & IgG1k-human & PD-L1 \\
\hline Tremelimumab & No brand name yet & CP-675,206 & Medimmune/AstraZeneca & IgG2-human & CTLA-4 \\
\hline Avelumab & Bavencio ${ }^{\circledR}$ & MSB0010718C & Merck KGaA & IgG1-human & PD-L1 \\
\hline Atezolizumab & Tecentriq ${ }^{\circledR}$ & MPDL3280A & Genentech/Roche & IgG1-humanized & PD-L1 \\
\hline Relatlimab & No brand name yet & BMS-986016 & Bristol-Myers Squibb & IgG4-human & LAG-3
\end{tabular}

sion of up to $20 \%$ is called "unconfirmed progressive disease" and should be verified by a second CT scan within 4-8 weeks so that it can be confirmed.

Pseudo-progression under immunotherapy has been described in some oncological diseases, including high-grade glioblastomas, non-small-cell lung carcinoma (NSCLC), and melanoma [3]. In this report, we present a case with an initial tumor progression after three cycles of pembrolizumab treatment that disappeared after 5 months, indicating the importance of the careful re-evaluation of treatment discontinuation of an immune checkpoint inhibitor, since longterm durable immunological effects might be possible. To our knowledge, this is the first description of a case with pseudo-progression after immunotherapy in gastroesophageal cancer.

\section{Immune checkpoint inhibitors and upper-GI tu- mors}

Upper-GI cancer, which includes gastric cancer, esophageal cancer, and gastroesophageal junction cancer, poses a major health problem and challenge worldwide, being the fourth most frequent cancer type (behind lung, breast, and colorectal cancer) and the second leading cause of cancer-related mortality worldwide (738,000 deaths annually; [4]). Despite the recent advances in treatment of upper-GI tumors, most patients live around 1 year in a metastatic setting. So far, attempts to establish novel treatments have shown that blocking human epidermal growth factor receptor 2 (HER2) with the monoclonal antibody trastuzumab is the only targeted therapy in the first-line (1L) setting.

Recently, The Genome Cancer Atlas (TGCA) came up with a new classification, which divides gastric cancer not on the basis of the histologic criteria, but according to genomic characteristics. TGCA identifies four major genomic subtypes: Epstein-Barr virus (EBV), microsatellite instability (MSI), genomically stable (GS), and chromosomal instability (CIN; [5]). However, the clinical significance of these four variants remains largely unknown and needs to be elucidated. Nevertheless, there are clear data indicating that the majority of the EBV-positive group shows positivity for the immune checkpoint molecule programmed death-ligand 1 (PD-L1). Furthermore, the association between MSI and response to immunotherapy is well known [6].

Taken together, there is a rationale for investigating novel immunotherapies such as cytotoxic t-lymphocyte-associated protein 4 (CTLA-4), PD-L1, and programmed cell death protein 1 (PD-1) inhibitors in upper-GI tumors. In this review, we analyze and evaluate different treatment approaches with immune checkpoint inhibitors in upper-GI cancers and provide an extensive overview of closed and ongoing clinical trials (see Tables 1 and 2).

\section{Pembrolizumab}

Pembrolizumab is an anti-PD-1 antibody that was investigated in advanced upper-GI tumors in the phase Ib Keynote-012 trial. In this trial, 39 PD-L1positive patients received pembrolizumab $10 \mathrm{mg} / \mathrm{kg}$ once every 2 weeks (Q2W). Eight patients (22\%) were assumed to have an overall response at the central review. Five patients $(13 \%)$ had a total of six grade 3 or 4 treatment-related adverse events (TRAE), including fatigue, pemphigoid, hypothyroidism, peripheral sensory neuropathy, and pneumonitis [7].

Based on these encouraging outcomes, the phase II Keynote-059 trial was conducted within the same patient population. It consisted of three cohorts. Cohort 1 included 259 patients who were treated with pembrolizumab alone as $\geq 3 \mathrm{~L}$. The overall response rate (ORR) was $11.6 \%$ and the median duration of response (DOR) was 8.4 months. Patients with PD-L1 ${ }^{+}$ cancer had an ORR of $15.5 \%$ and a median DOR of 16.3 months. By contrast, patients with PD-L1- tumors achieved an ORR of only $6.4 \%$ and a median DOR of approximately 7 months. Grade $\geq 3$ TRAE were documented in $18 \%$ of patients (46 patients).

In cohort 2, 764 patients with HER2-negative, PD- $1^{+}$, relapsed or metastasized upper-GI tumors were given pembrolizumab $200 \mathrm{mg}+$ cisplatin $80 \mathrm{mg} / \mathrm{m}^{2}+5$-FU $\quad 800 \mathrm{mg} / \mathrm{m}^{2}$ (or capecitabine $1000 \mathrm{mg} / \mathrm{m}^{2}$ in Japan) for six cycles once every 3 weeks (Q3W) for up to 2 years or until disease progression, the patient's/investigator's decision to withdraw, or unacceptable toxicity. The median OS was 13.6 months, the median PFS was 6.6 months, and the 


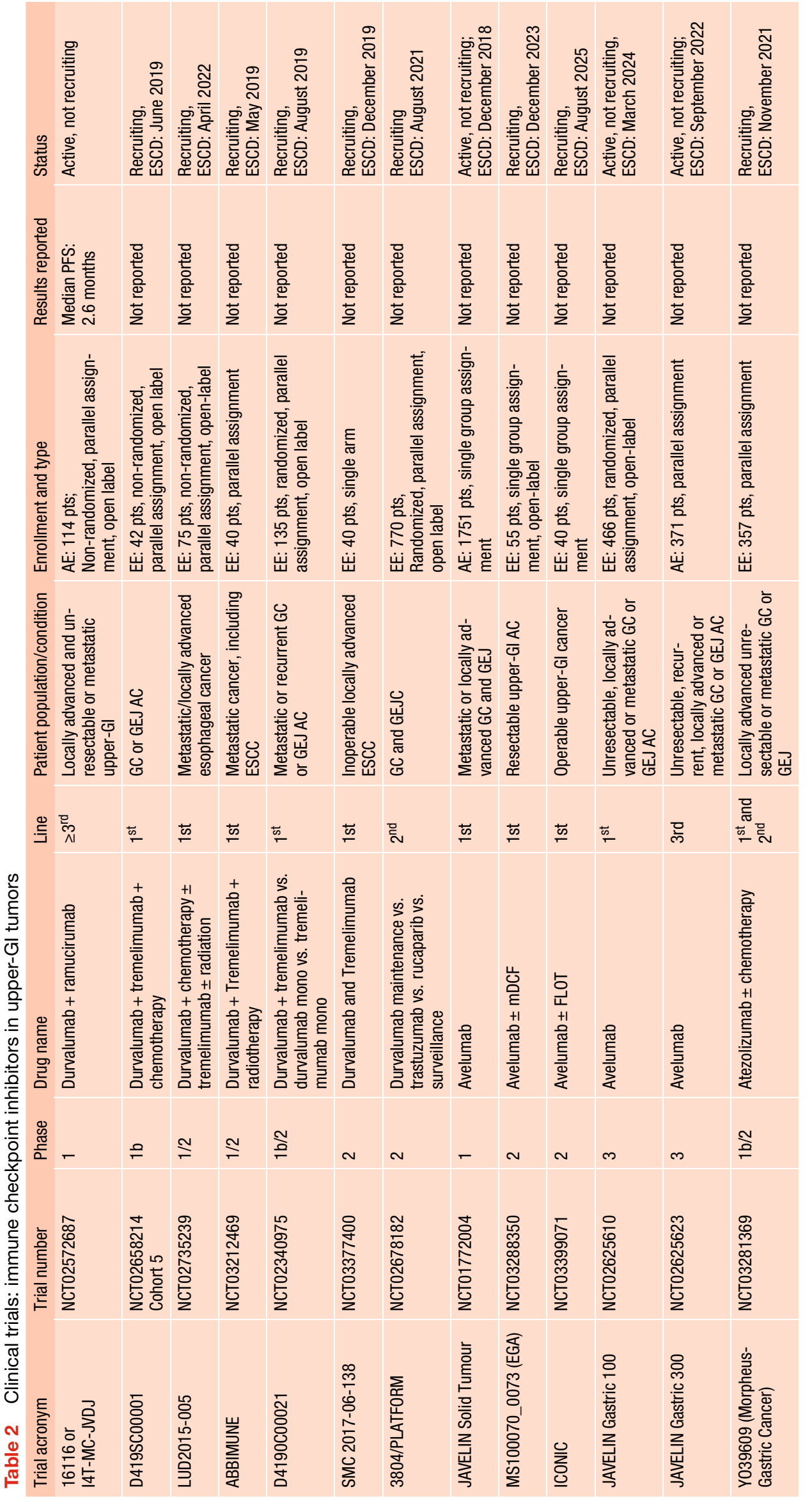




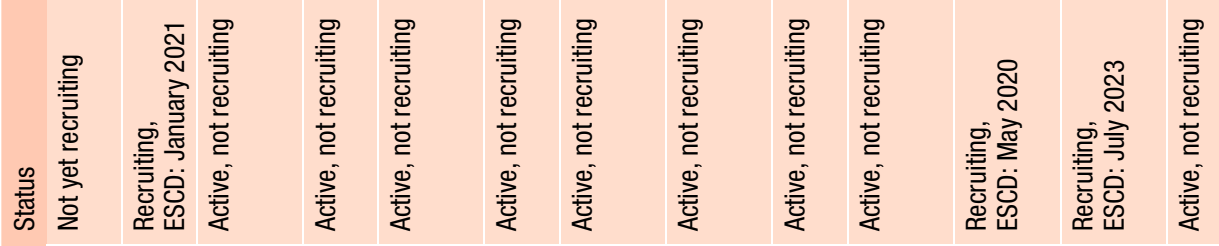

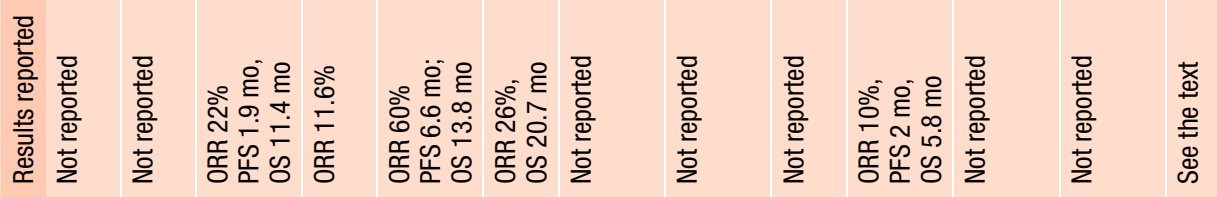

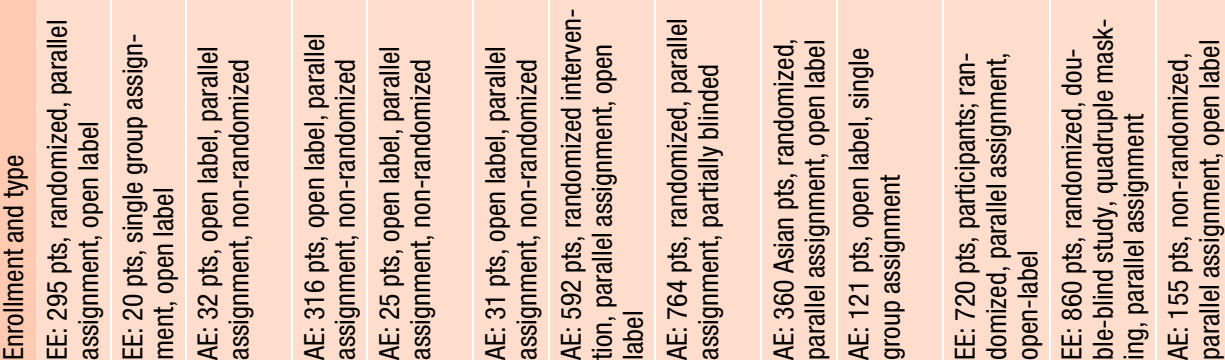

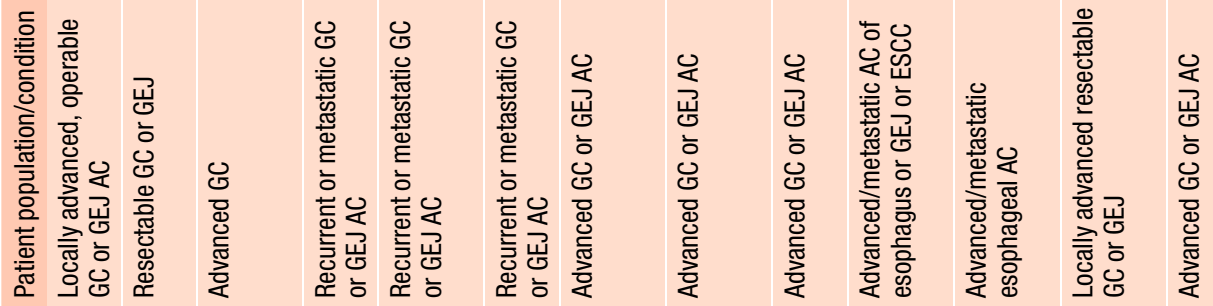

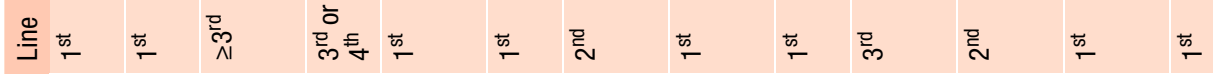

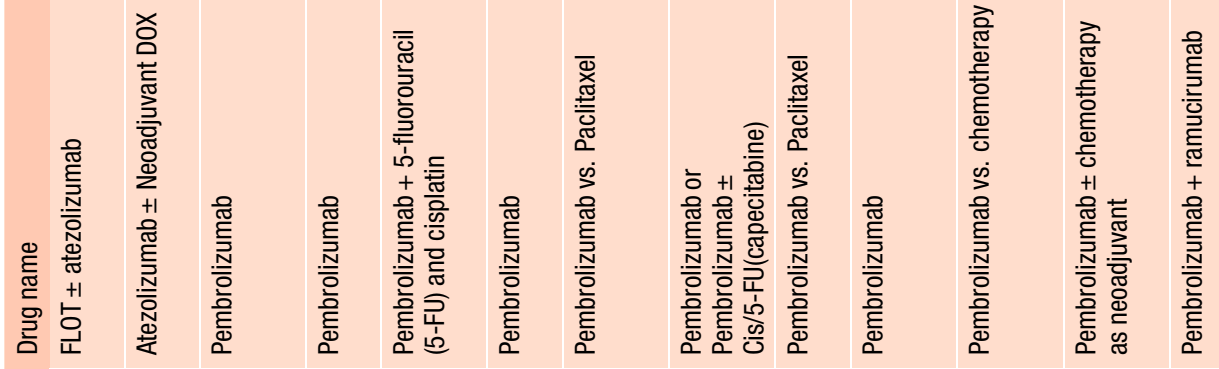

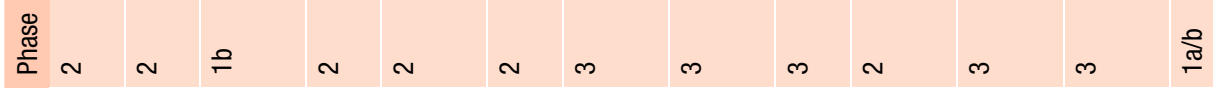

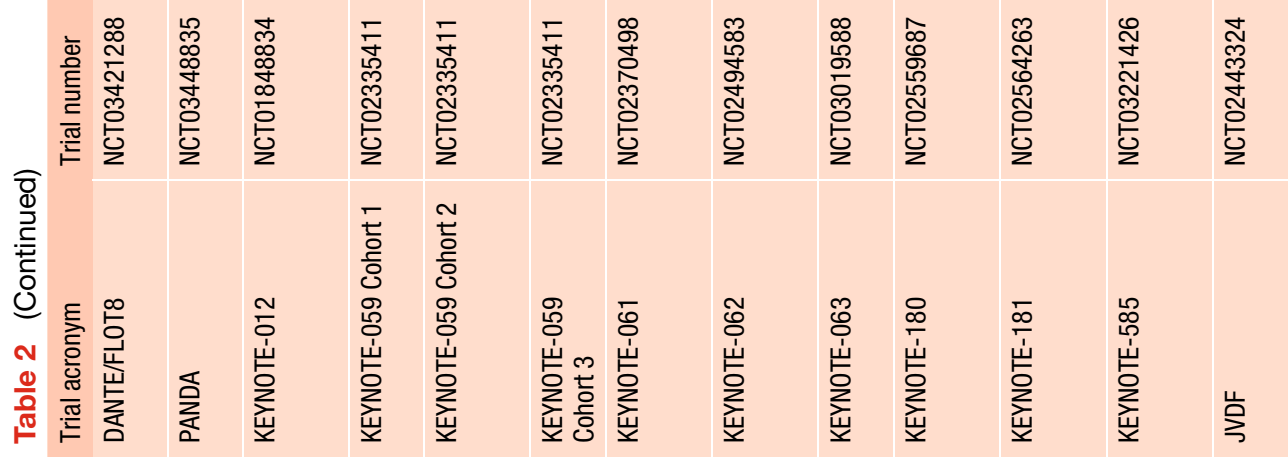




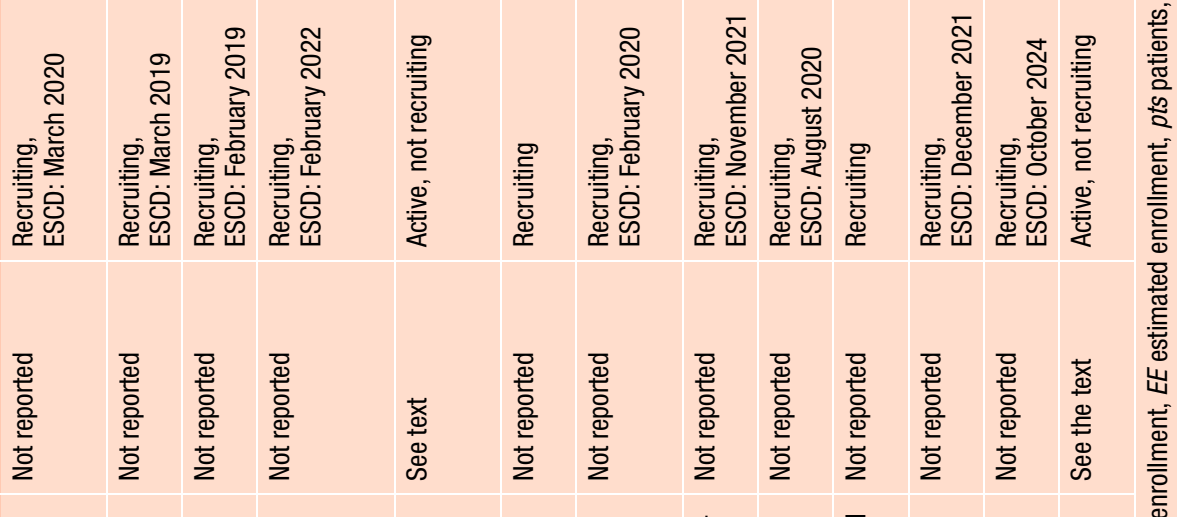

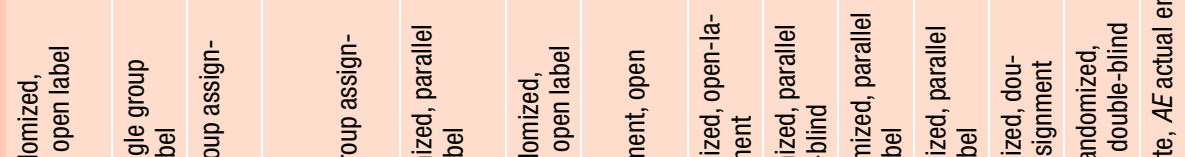

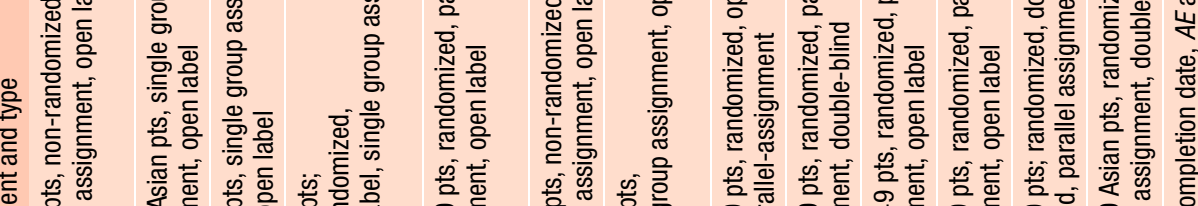

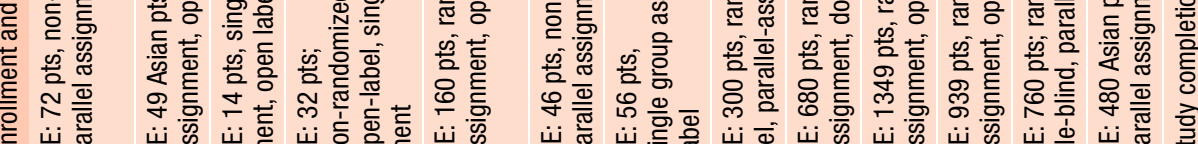

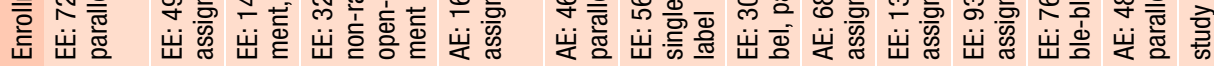

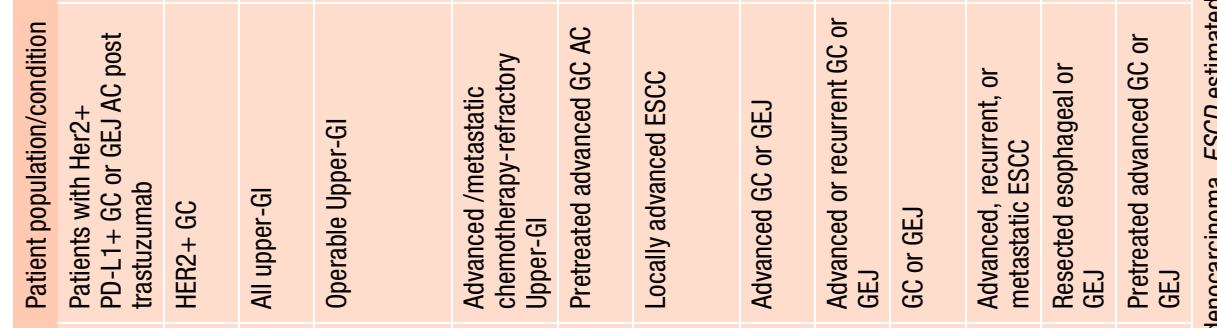

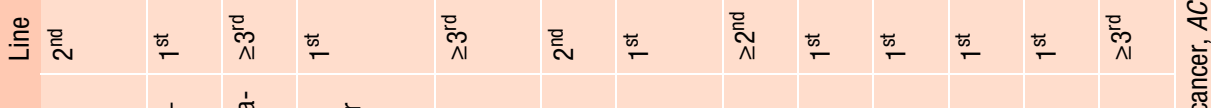

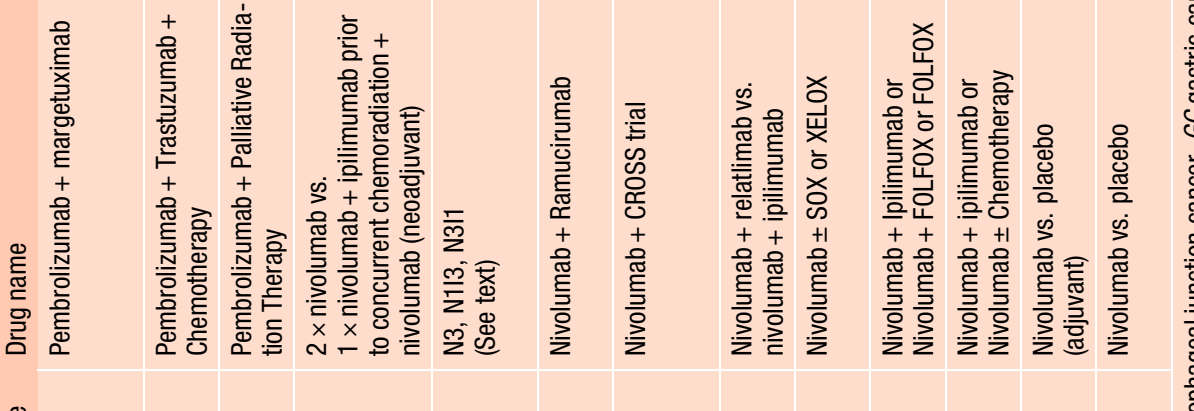

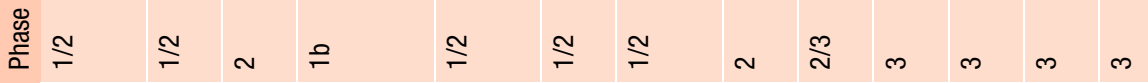

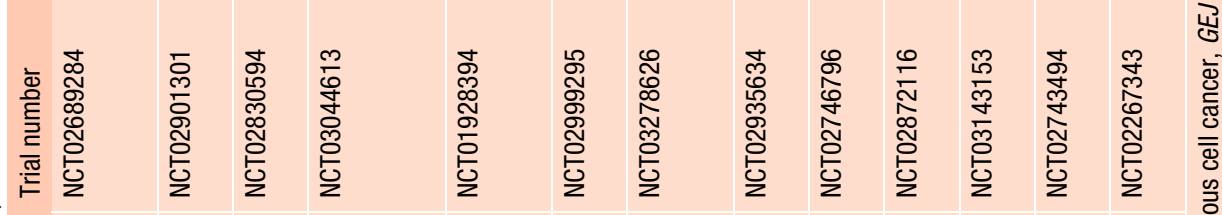

宇

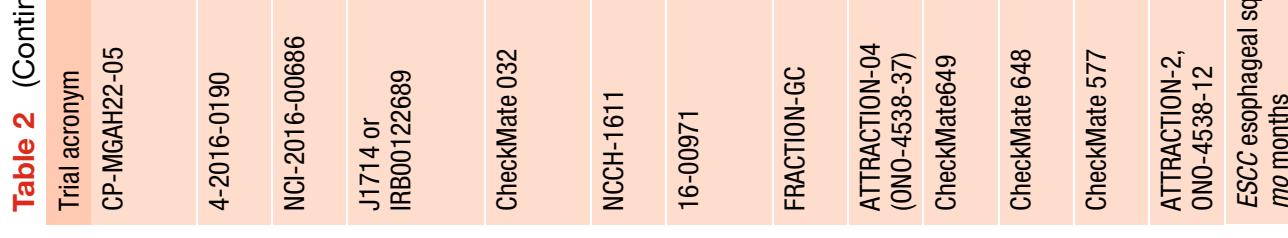


ORR was $60 \%$. However, the ORR in PD-L1+ tumors was $69 \%$, over $30 \%$ higher than in $\mathrm{PD}-\mathrm{L1}^{-}$tumors $(37.5 \%)$. At the time of writing this report, we do not have the data on the OS rates based on the PD-L1 status. It will be interesting to see whether the higher ORR is translated into extended OS in the PD-L1+ group.

In the third cohort of the Keynote-059 trial, pembrolizumab mono was administered as frontline treatment in 31 patients with HER2-/PD-L1 ${ }^{+}$upper-GI tumors. In this setting, pembrolizumab yielded an ORR of only $26 \%$, which is much lower than the expected ORR of conventional chemotherapy (40\%). Interestingly, despite the lower ORR value, the median OS was remarkable at 20.7 months. Grade $\geq 3$ TRAE occurred in $23 \%$ of patients [8].

Angiogenesis and immunosuppression are hallmarks of tumor growth. Therefore, the activity and the safety of pembrolizumab in combination with the anti-angiogenic therapy ramucirumab were assessed in a phase Ia/b trial (NCT02443324) in patients with prior progression on systemic therapy. Ramucirumab was applied at $8 \mathrm{mg} / \mathrm{kg}$ on days 1 and 8 with pembrolizumab $200 \mathrm{mg}$ Q3W. In mid-2017, preliminary results were reported for 28 patients, of whom $68 \%$ were $\mathrm{PD}-\mathrm{L1}^{+}$and approximately $75 \%$ were male. In total, $61 \%$ of the patients suffered from grade 3 TRAE; however, grade 4/5 TRAE was not reported. PFS was 5.3 months. An objective response was attained in $25 \%$ of the patients, six of whom (21\% of all patients) were $\mathrm{PD}-\mathrm{Ll}^{+}[9]$.

In the phase II Keynote-180 study, 121 heavily pretreated patients with gastroesophageal cancer were included. Approximately $50 \%$ of them were PD-L1 ${ }^{+}$ and $83 \%$ were male; $200 \mathrm{mg}$ pembrolizumab alone was given $\mathrm{Q} 3 \mathrm{~W}$ as a third-line (3L) treatment for $35 \mathrm{cy}-$ cles and achieved a $10 \%$ ORR and a median OS of 5.8 months [10].

As a further step, a phase III study was designed where pembrolizumab in combination with $1 \mathrm{~L}$ therapy for advanced upper-GI cancer was administered (Keynote-062/NCT02494583). In this study, 764 patients with locally advanced/metastatic PD-L1+/HER2gastric or GEJ adenocarcinoma were included. Patients were randomized $1: 1: 1$ to pembrolizumab $200 \mathrm{mg}$ Q3W (arm 1), pembrolizumab + cisplatin Q3W + 5-fluorouracil (5-FU) on days 1-5 of each Q3W cycle (arm 2), or a placebo Q3W + cisplatin +5-FU (arm 3) [11].

Two more phase III trials, Keynote-061 and Keynote-063, which compared pembrolizumab versus paclitaxel as a second-line (2L) treatment, were conducted in non-Asian and Asian patients, respectively. However, Keynote-061 failed to meet its primary endpoints of a superior OS and PFS for pembrolizumab versus paclitaxel $[12,13]$.

Another study included patients with $\mathrm{HER}^{+}$gastric or GEJ cancer after $1 \mathrm{~L}$ treatment. This phase $\mathrm{Ib} / \mathrm{II}$ study evaluated pembrolizumab in combination with margetuximab, an HER2 antibody with a more affine Fc domain for the activation of CD16A Fc receptors on NK cells. The preliminary results show an ORR of $18.4 \%$. A notably higher ORR $(35.7 \%)$ was reported in Asian patients than in American patients (8.3\%; [14]).

\section{Nivolumab}

The phase I/II CheckMate-032 trial evaluated the clinical activity of nivolumab with or without ipilimumab in Western patients as a $\geq 3 \mathrm{~L}$ treatment. It included 160 patients who were divided into three groups: 59 patients received $3 \mathrm{mg} / \mathrm{kg}$ nivolumab Q2W (N3), 49 patients were given nivolumab $1 \mathrm{mg} / \mathrm{kg}+$ ipilimumab $3 \mathrm{mg} / \mathrm{kg}$ Q3W $(\mathrm{N} 1+\mathrm{I} 3)$, and 52 patients received nivolumab $3 \mathrm{mg} / \mathrm{kg}+$ ipilimumab $1 \mathrm{mg} / \mathrm{kg} \quad$ Q3W $(\mathrm{N} 3+\mathrm{I} 1)$. Approximately $25 \%$ of all the patients had a PD-L1 ${ }^{+}$status. The ORR was $12 \%$ in the N3 group, $24 \%$ in the $\mathrm{N} 1+\mathrm{I} 3$ group, and $8 \%$ in the $\mathrm{N} 3+\mathrm{I} 1$ group. However, the ORR in $\mathrm{PD}-\mathrm{Ll}^{+}$patients was higher than in $\mathrm{PD}-\mathrm{L1}^{-}$patients. The median OS was 6.2 months in the N3, 6.9 months in the N3+I1, and 4.8 months in the N3+I1 groups. Patients with both PD-L1-positive and -negative status benefitted from the treatment; however, positive patients had higher ORR. Patients with $\mathrm{PD}-\mathrm{L} 1 \geq 1 \%$ cancer had an ORR of $19 \%$ in the N3, $40 \%$ in the $\mathrm{N} 1+\mathrm{I} 3$, and $23 \%$ in the $\mathrm{N} 1+\mathrm{I} 3$ groups, whereas the ORR was $12 \%, 22 \%$, and $10 \%$, respectively, in patients with $\mathrm{PD}-\mathrm{L} 1<1 \%$ cancer [15].

Nivolumab as salvage treatment after $2 \mathrm{~L}$ or laterline chemotherapy in advanced disease was tested in a phase III trial (NCT02267343/ATTRATCTION2). In this trial, 493 patients were randomized to receive $3 \mathrm{mg} / \mathrm{kg}$ nivolumab $(n=330)$ or a placebo ( $n=163)$ Q2W. The median OS was 5.26 months with nivolumab and 4.14 months with the placebo, and the OS rates at 6 and 12 months were $46.4 \%$ versus $34.7 \%$ and $26.6 \%$ versus $10.9 \%$, respectively. The ORR was $11.2 \%$ with nivolumab versus $0 \%$ with the placebo. The median PFS was 1.61 months with nivolumab versus 1.45 months with the placebo. Grade $\geq 3$ TRAE occurred in $11.5 \%$ with nivolumab and $5.5 \%$ with the placebo [16].

\section{Other immune checkpoint inhibitors}

\section{Avelumab}

The safety and clinical activity of avelumab as a $1 \mathrm{~L}$ maintenance or 2L therapy were tested in advanced disease (NCT01772004). Patients received avelumab at $10 \mathrm{mg} / \mathrm{kg}$ IV Q2W. TRAEs of any grade occurred in 89 patients $(58.9 \%)$. In total, 14 patients had an unconfirmed response: in 2L, six out of 62 (9.7\%), all partial response (PRs); in maintenance treatment, eight out of $89(9.0 \%)$ due to two complete response (CRs) and six PRs. In $2 \mathrm{~L}$ and maintenance patients, the median PFS was 6 weeks and 12 weeks, respectively [17].

Motivated by these facts, an open-label phase III study (NCT02625610 JAVELIN Gastric 100) compared 
maintenance treatment with single-agent avelumab versus continuation of $1 \mathrm{~L}$ chemotherapy in patients with GC/GEJC [18].

The JAVELIN Gastric 300 trial included heavily pretreated patients with upper-GI tumors and administered either avelumab or the investigator's choice of chemotherapy. According to the recent press release, the primary endpoint (OS) of this trial has not been reached [19].

\section{Atezolizumab}

NCT03281369 consists of two cohorts: One cohort includes patients who experienced progressive disease after platinum-containing or fluoropyrimidinecontaining chemotherapy in $1 \mathrm{~L}$ treatment and the other cohort consists of patients who will receive atezolizumab as a 1L treatment [20].

In the phase II FLOT8 trial, FLOT chemotherapy is either given alone (arm B) or in combination with atezolizumab ( $\operatorname{arm~A}$ ) as a frontline treatment in patients with a resectable upper-GI tumor [21].

\section{Durvalumab \pm Tremelimumab}

A recently published phase I/II trial showed a manageable safety profile for durvalumab mono in over 50 pretreated upper-GI patients and proved its efficacy with a median OS of 4.9 months (NCT01693562; [22]).

In the single-arm phase II NCT03377400 trial, two cycles of 5-FU/cisplatin are applied together with durvalumab + tremelimumab Q3W with concurrent radiotherapy for inoperable locally advanced esophageal squamous cell carcinoma. After this therapy, two cycles of durvalumab + tremelimumab are given QW4 followed by durvalumab monotherapy Q4W for a maximum of 2 years of enrolment [23].

Furthermore, the efficacy of durvalumab in combination with tremelimumab is compared against durvalumab monotherapy or tremelimumab monotherapy in upper-GI tumors [24].

\section{Discussion of the review}

Immune checkpoint inhibitors are well tolerated and have a manageable safety profile. They showed some effectivity in several large trials in patients with gastroesophageal cancer and achieved an ORR of at least $10 \%$ and an OS of at least 5 months in a salvage setting. Based on cohort 1 of the Keynote- 059 trial, pembrolizumab was approved for $\mathrm{PD}-\mathrm{Ll}^{+}$, chemotherapyrefractory upper-GI tumors by the FDA. Similarly, the results of the ATTRACTION-2 trial led to the approval of nivolumab in Japan in biomarker-unselected, heavily pretreated upper-GI tumor patients.

There is a tendency for pembrolizumab trials to show more benefit for $\mathrm{PD}-\mathrm{Ll}^{+}$patients, whereas nivolumab seems to be equally effective in both $\mathrm{PD}_{-\mathrm{L}^{+}}{ }^{+}$and $\mathrm{PD}-\mathrm{L1}^{-}$patients. Although not discussed in detail in this review, the methodology of the PD-
L1 measurements shows a great variety for individual drugs and there is a significant lack of standardization of the assessment.

Drugs such as pembrolizumab, nivolumab, and avelumab are tested mainly in salvage settings, and the results of large-scale trials of first- and second-line setting are eagerly awaited. It would be interesting to ascertain the benefit and efficacy of the combination of immune checkpoint inhibitors with other anti-tumor strategies including chemotherapy and radiation therapy. Another interesting aspect is the combination of two different immunotherapy reagents or the combination with other targeted therapies including anti-HER2, anti-angiogenesis, or tyrosine-kinase inhibitors.

\section{Take-home message}

Immune checkpoint inhibitors may induce clinically significant responses in advanced upper-Gl tumors. Promising data from large clinical trials evaluating this treatment approach in this setting are emerging.

Funding Open access funding provided by Medical University of Vienna.

Conflict of interest H. Taghizadeh, K. Lampichler, A. Beer, M. Preusser, and A. Ilhan-Mutlu declare that they have no competing interests.

Open Access This article is distributed under the terms of the Creative Commons Attribution 4.0 International License (http://creativecommons.org/licenses/by/4.0/), which permits unrestricted use, distribution, and reproduction in any medium, provided you give appropriate credit to the original author(s) and the source, provide a link to the Creative Commons license, and indicate if changes were made.

\section{References}

1. Eisenhauer EA, Therasse P, Bogaerts J, et al. New response evaluation criteria in solid tumours: revised RECIST guideline (version 1.1). Eur JCancer. 2009;45(2):228-47.

2. Seymour L, Bogaerts J, Perrone A, et al. iRECIST: guidelines for response criteria for use in trials testing immunotherapeutics. Lancet Oncol. 2017;18(3):e143-e52.

3. Beer L, Hochmair M, Prosch H. Pitfalls in the radiological response assessment of immunotherapy. Memo. 2018;11(2):138-43.

4. Sitarz R, Skierucha M, Mielko J, et al. Gastric cancer: epidemiology, prevention, classification, and treatment. Cancer Manag Res. 2018;10:239-48.

5. Cancer Genome Research AN. Comprehensive molecular characterization of gastric adenocarcinoma. Nature. 2014;513(7517):202-9.

6. Leal AD, Paludo J, Finnes HD, Grothey A. Response to pembrolizumab in patients with mismatch repair deficient (dMMR) colorectal cancer (CRC). J Clin Oncol. 2017; https://doi.org/10.1200/jco.2017.35.15_suppl.3558.

7. Muro K, Chung HC, Shankaran V, et al. Pembrolizumab for patients with PD-L1-positive advanced gastric cancer (KEYNOTE-012): a multicentre, open-label, phase $1 \mathrm{~b}$ trial. LancetOncol. 2016;17(6):717-26. 
8. Fuchs CS, Doi T, Jang RW, et al. Safety and efficacy of pembrolizumab monotherapy in patients with previously treated advanced gastric and gastroesophageal junction cancer: phase 2 clinical KEYNOTE-059 trial. JAMA Oncol. 2018;4(5):e180013.

9. Chau I, Bendell JC, Calvo E, Santana-Davila R, et al. Interim safety and clinical activity in patients (pts) with advanced gastric or gastroesophageal junction (G/GEJ) adenocarcinoma from a multicohort phase 1 study of ramucirumab (R) plus pembrolizumab (P). JClin Oncol. 2017;35:102. https:// doi.org/10.1200/jco.2017.35.4_suppl.102.

10. Shah MA, Bennouna J, Shen L, Enzinger PC, et al. Pembrolizumab (MK-3475) for previously treated metastatic adenocarcinoma or squamous cell carcinoma of the esophagus: Phase II KEYNOTE-180 study. J Clin Oncol. 2016;34(4):suppl. https://doi.org/10.1200/jco.2016.34.4_ suppl.tps 189

11. Tabernero J, Bang Y-J, Fuchs CS, Ohtsu A, et al. KEYNOTE062: Phase III study of pembrolizumab (MK-3475) alone or in combination with chemotherapy versus chemotherapy alone as first-line therapy for advanced gastric or gastroesophageal junction (GEJ) adenocarcinoma. J Clin Oncol. 2017;34:4_suppl. https://doi.org/10.1200/jco.2016.34.4_ suppl.tps185.

12. Shitara K, Ozguroglu M, BangYJ, DiBartolomeo M, Mandala M, Ryu MH, Fornaro L, Olesinski T, Caglevic C, Chung HC, et al. Pembrolizumab versus paclitaxel for previously treated, advanced gastric or gastro-oesophageal junction cancer (KEYNOTE-061): a randomised, open-label, controlled, phase 3 trial. Lancet. 2018;392(10142):123-33.

13. Efficacy and Safety Study of Pembrolizumab (MK-3475) Versus Paclitaxel in Asian Participants With Advanced Gastric or Gastroesophageal Junction Adenocarcinoma Who Progressed After First-line Therapy With Platinum and Fluoropyrimidine (MK-3475-063/KEYNOTE-063) [https:// clinicaltrials.gov/ct2/show/NCT03019588]. Accessed: 15 Nov2018.

14. Catenacci DVT, Park H, Lockhart AC, Gold PJ, Enzinger $P C$ et al. Phase $1 \mathrm{~b} / 2$ study of margetuximab (M) plus pembrolizumab (P) in advanced HER2+ gastroesophageal junction (GEJ) or gastric (G) adenocarcinoma (GEA). J Clin Oncol. 2018;36(4):140-140.

15. Yuriy Janjigian Y, Ott PA, Calvo E, Kim JW, et al. Nivolumab \pm ipilimumab in pts with advanced $(\mathrm{adv}) /$ metastatic chemotherapy-refractory $(\mathrm{CTx}-\mathrm{R})$ gastric $(\mathrm{G})$, esophageal (E), or gastroesophageal junction (GEJ) cancer: CheckMate 032 study. J Clin Oncol. 2017; https://doi.org/10.1200/jco. 2017.35.15_suppl.4014.

16. Kang YK, Boku N, Satoh T, et al. Nivolumab in patients with advanced gastric or gastro-oesophageal junction cancer refractory to, or intolerant of, at least two previous chemotherapy regimens (ONO-4538-12, ATTRACTION-2): a randomised, double-blind, placebo-controlled, phase 3 trial. Lancet. 2017;390(10111):2461-71.
17. Chung HC, Arkenau H-T, WyrwiczL, OhD-Y, etal. Avelumab (MSB0010718C; anti-PD-L1) in patients with advanced gastric or gastroesophageal junction cancer from JAVELIN solid tumour phase Ib trial: Analysis of safety and clinical activity. J Clin Oncol. 2016;34(15):4009-4009. https://doi. org/10.1200/JCO.2016.34.15_suppl.4009

18. Moehler M, Ryu M-H, Lee K-W, Coskun HS, et al. JAVELIN Gastric 100: Phase 3 trial of avelumab (anti-PD-L1) maintenance therapy versus continuation of first-line chemotherapy in patients with advanced gastric or gastroesophageal junction cancer (GC/GEJC). J Clin Oncol. 2018;36(4). https://doi.org/10.1200/JCO.2018.36.4_suppl.TPS195

19. Pfizer MKa: Update on Phase III JAVELIN Gastric 300 Trial of Avelumab in Pretreated Advanced Gastric Cancer. 2017. https://investors.pfizer.com/ investor-news/press-release- details/2017/Merck-KGaADarmstadt-Germany-and-Pfizer-Provide-Update- onPhase-III-JAVELIN-Gastric-300-Study-in-Patients-withPre-Treated-Advanced-Gastric-Cancer/default.aspx

20. Oh D-Y, Al-Batran S-E, Kim K-P, Abbas Manji G, et al. MORPHEUS: A phase Ib/II multi-trial platform evaluating the efficacy and safety of cancer immunotherapy (CIT)-based combinations in patients (pts) with gastric or pancreatic cancer. 2018;36(15). https://doi.org/10.1200/JCO.2018.36. 15_suppl.TPS4134

21. Phase II Study of Atezolizumab + FLOT vs. FLOT Alone in Patients With Gastric Cancer and GEJ (DANTE). https:/ / clinicaltrials.gov/ct2/show/NCT03421288. Accessed: 15 Nov2018.

22. Hollebecque A, Wainberg ZA, Ajani JA, Marshall J, et al. Safety and clinical activity of durvalumab monotherapy in patients with gastroesophageal cancers. J Clin Oncol. 2018;36(15):4032-4032. https://doi.org/10.1200/JCO. 2018.36.15_suppl.4032.

23. Definitive CCRT Combined With Durvalumab and Tremelimumab for Inoperable Esophageal Cancer [https:// clinicaltrials.gov/ct2/show/NCT03377400]. Accessed: 15 Nov2018.

24. A Phase 1b/2 Study of MEDI4736 With Tremelimumab, MEDI4736 or Tremelimumab Monotherapy in Gastric or GEJAdenocarcinoma [https://clinicaltrials.gov/ct2/show/ NCT02340975]. Accessed: 15 Nov 2018.

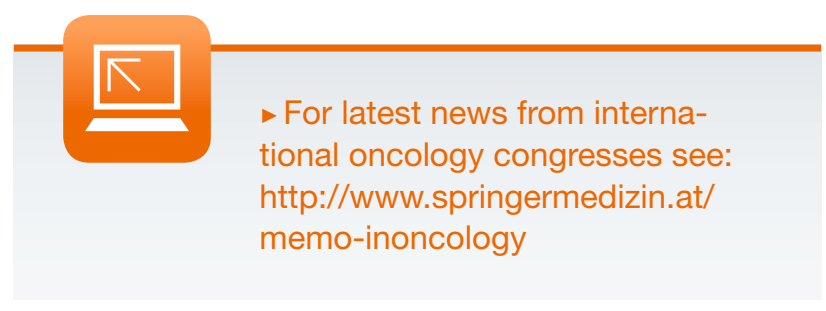

